ceftriaxone with other antibiotics leads to faster clearance. TOC for pharyngeal $\mathrm{Ng} 7$ days after treatment may be too soon.

Support: This study was funded by Excellence Scholarship Program (Beasiswa Unggulan), Ministry of Research, Technology and Higher Education, Republic of Indonesia and Public Health Service of Amsterdam, the Netherlands

\subsection{A PHASE II, RANDOMISED, STUDY IN ADULT SUBJECTS EVALUATING THE EFFICACY, SAFETY, AND TOLERABILITY OF SINGLE DOSES OF GEPOTIDACIN (GSK2140944) FOR TREATMENT OF UNCOMPLICATED UROGENITAL GONORRHOEA}

${ }^{1}$ Caroline Perry, ${ }^{2}$ Etienne Dumont, ${ }^{2}$ Caroline Perry, ${ }^{2}$ Aparna Raychaudhuri. 'Louisiana State University Health Sciences Centre, New Orleans, La, USA; ${ }^{2}$ Glaxosmithkline, Collegeville, Pa, USA

\subsection{6/sextrans-2017-053264.26}

Introduction Gonorrhoea is currently the second most common bacterial sexually transmitted infection and represents a serious public health threat. The increasing antimicrobial resistance in Neisseria gonorrhoeae (GC) to currently available therapies is driving an urgent need for new novel agents. Gepotidacin (GEP) is a novel, first in class triazaacenaphthylene antibacterial which inhibits bacterial DNA replication. This multicenter (11 US and 1 UK) trial evaluated GEP as a single oral dose in men and women.

Methods Patients with signs and symptoms of urogenital gonorrhoea, a prior culture or nucleic acid amplification test (NAAT) positive for GC, a urethral Gram stain with intracellular diplococci, or who had sexual contact with an individual diagnosed with gonorrhoea in the past 14 days were eligible for enrollment. Participants were randomised 1:1 to receive either $1.5 \mathrm{~g}$ or $3 \mathrm{~g}$ GEP orally. The primary efficacy endpoint was culture confirmed microbiological eradication at test-ofcure (TOC) visit 3-7 days post dose.

Results 106 patients (101 men and 5 women) were randomised and 105 received treatment. Baseline GC isolates were identified in $69(65 \%)$ urogenital, $3(3 \%)$ pharyngeal, and 4 (4\%) rectal specimens. Microbiological success was achieved by $97 \%$ and $95 \%$ of subjects with urogenital GC in the $1.5 \mathrm{~g}$ and $3 \mathrm{~g}$ treatment groups, respectively. Isolates from 2 subjects developed resistance to GEP between baseline and TOC. The most common GEP-related AEs were gastrointestinal (diarrhoea, flatulence, abdominal pain and nausea) with the majority being mild or moderate in intensity. Treatment-related AEs of moderate intensity occurred with a higher incidence in the $3 \mathrm{~g}$ treatment group than the $1.5 \mathrm{~g}$ treatment group $(15 \%$ and $10 \%$, respectively). There were no AEs that led to study withdrawal and no SAEs were reported.

Conclusions Both the GEP $1.5 \mathrm{~g}$ and $3 \mathrm{~g}$ single doses eradicated urogenital GC with microbiological success rates of 29/30 (97\%) and 37/39 (95\%), respectively. The data support further development of GEP in this indication.

Support: This study was supported by GSK (BTZ116576; NCT02294682). This project was funded in whole or in part with Federal funds from the Office of the Assistant Secretary for Preparedness and Response, Biomedical Advanced. Research and Development Authority (BARDA), under agreement \# HHSO100201300011C

\subsection{DOES HIGH-LEVEL AZITHROMYCIN RESISTANCE EMERGE FROM LOW-LEVEL RESISTANCE IN NEISSERIA GONORRHOEAE?}

${ }^{1}$ Michelle Cole, ${ }^{1}$ Anthony Underwood, ${ }^{2}$ Kate Templeton, ${ }^{2} \mathrm{Jill}$ Shepherd, 'Gwenda Hughes, ${ }^{1}$ Nazim Mustafa, ${ }^{1}$ Francesco Tripodo, ${ }^{1}$ Neil Woodford, ${ }^{1}$ Helen Fifer. ${ }^{1}$ National Infections Service, London, UK; ${ }^{2}$ Scottish Bacterial Sexually Transmitted Infections Reference Laboratory, Edinburgh, UK

\subsection{6/sextrans-2017-053264.27}

Introduction High-level azithromycin (Azi) resistance (HL-AziR) threatens gonorrhoea dual therapy (ceftriaxone $500 \mathrm{mg}$ and Azi 1g) as it renders Azi ineffective. Between November 2014-2016, 58 cases of HL-AziR (MIC >256 mg/L) N. gonorrhoeae (NG) were detected in England. Whole genome sequencing (WGS) revealed that most HL-AziR isolates were from a single clade (NG-MAST ST9768) with an A2059G mutation in $3 / 4$ or all 4 alleles of the $23 \mathrm{~S}$ rRNA gene. Lower-level AziR (MICs $1.0-32 \mathrm{mg} / \mathrm{L}$ ) is commonly associated with a C2611T 23S rRNA gene mutation and $m t r R$ promoter mutations. We performed WGS of ST9768 isolates with Azi susceptibility (MICs).

Methods WGS was performed on 7 non-HL-AziR ST9768 isolates from Scotland isolated in 2014. A phylogeny was constructed using the maximum likelihood algorithm based on whole genome variants. Genetic resistance determinants were analysed by mapping the WGS short reads to the $23 \mathrm{~S}$ rRNA gene.

Results All ST9768 isolates with Azi MICs of $0.12-1.0 \mathrm{mg} / \mathrm{L}$ were part of the same WGS clade as the ST9768 HL-AziR isolates. One susceptible isolate (MIC $0.12 \mathrm{mg} / \mathrm{L}$ ) had $0 / 4$ mutated (A2059G) 23S rRNA alleles, five susceptible isolates (MICs $0.25 \mathrm{mg} / \mathrm{L}$ ) had $1 / 4$ mutated alleles and one low-level resistant isolate (MIC $1.0 \mathrm{mg} / \mathrm{L}$ ) had $2 / 4$ mutated alleles. No isolates carried the $\mathrm{C} 2611 \mathrm{~T}$ mutation.

Conclusion This is the first report of the A2059G mutation in NG isolates with Azi MICs of $0.25-1.0 \mathrm{mg} / \mathrm{L}$. The phylogeny suggested that the HL-AziR ST9768 isolates are descendants of the low-level AziR isolates, which are in turn, descendants of the susceptible isolates. We hypothesise that azithromycin exposure provided selection pressure for one or two mutated copies of the $23 \mathrm{~S}$ rRNA gene to recombine with wild-type copies, leading to 3 to 4 mutated copies in HL-AziR isolates. Greater understanding of the prevalent mechanisms of lower level AziR is required as HL-AziR could emerge in isolates with A2059 mutations and eliminate the effectiveness of dual therapy.

\subsection{WHAT ROLE DOES IMPORTATION PLAY IN THE SPREAD OF ANTIMICROBIAL RESISTANT NEISSERIA GONORRHOEAE IN THE UK? ASSOCIATIONS BETWEEN ANTIMICROBIAL RESISTANT STRAINS AND RECENT SEX ABROAD}

${ }^{1}$ Katy Town, ${ }^{2}$ Nigel Field, ${ }^{3}$ Michelle Cole, ${ }^{4}$ Simon Harris, ${ }^{3}$ Gwenda Hughes. ${ }^{7}$ NIHR HPRU in Blood Borne and Sexually Transmitted Infections, UCL, London, UK; ${ }^{2}$ Research Department of Infection And Population Health, Ucl, London, UK; ${ }^{3}$ National Infections Service, Public Health England, London, UK; ${ }^{4}$ The Wellcome Trust Sanger Institute, Cambridge, UK

\subsection{6/sextrans-2017-053264.28}

Introduction People living in Britain who have sex abroad are more likely to report sexual behaviour that puts them at greater risk of acquiring STIs, including Neisseria gonorrhoeae 
(NG). Antimicrobial resistant (AMR) NG is a global public health concern, which may emerge de novo or be imported to the UK when individuals infected abroad have subsequent sexual partners at home. We investigated whether patients who reported sex outside the UK ('sex abroad') were more or less likely to be diagnosed with AMR NG.

Methods Logistic regression was used to model the association between reported recent sex abroad and decreased susceptibility (DS) to ceftriaxone (MIC $(\mathrm{mg} / \mathrm{L})>0.015)$ and cefixime (0.125) and azithromycin AMR (>1) stratifying by sexual orientation (men who have sex with men (MSM) and heterosexual men and women) from isolates in England and Wales collected within the Gonococcal Resistance to Antimicrobials Surveillance Programme, 2004-2015.

Results Over $10 \%$ of MSM and heterosexuals reported sex abroad. Among heterosexuals, infection with a strain of NG with DS to ceftriaxone was associated with sex abroad after adjusting for potential confounders: ceftriaxone (DS prevalence, adjusted odds ratio ( $95 \%$ confidence interval)): $14 \%$, 1.8 (1.3-2.3). Infection with NG DS/AMR to cefixime or azithromycin was not associated with reported sex abroad after adjusting for potential confounders: cefixime 4\%, 1.6 (0.92.7); azithromycin 2\%, 1.5 (0.7-3.3). For MSM, no association was found between infections with DS/AMR NG and sex abroad.

Conclusion In the UK, heterosexuals with NG infection who report sex abroad are at a higher risk of DS to ceftriaxone, suggesting that sex abroad might be the source of some AMR NG within heterosexual networks and highlighting the importance of condom use for travellers. In contrast, DS/AMR NG was not associated with sex abroad among MSM, which might reflect established AMR within MSM networks in the UK. Genetic comparison of these isolates using whole genome sequencing might further elucidate how AMR NG is imported and disseminated in the UK.

\subsection{THE IMPACT OF A RAPID GENOTYPIC NEISSERIA GONORRHOEAE ASSAY ON TARGETED CIPROFLOXACIN THERAPY}

${ }^{1}$ Lao-Tzu Allan-Blitz, ${ }^{2}$ Romney M Humphries, ${ }^{2}$ Peera Hemarajata, ${ }^{3}$ Ashima Bhatti, ${ }^{4}$ Jeffrey D Klausner. 'David Geffen School of Medicine, Ucla, Los Angeles, USA; ${ }^{2}$ Department of Laboratory Medicine, University of California LoS Angeles, LOS Angeles, USA; ${ }^{3}$ Fielding School of Public Health, University of California Los Angeles, Los Angeles, USA; ${ }^{4}$ Division of Infectious Diseases: Department of Medicine, University of California Los Angeles, Los Angeles, USA

\subsection{6/sextrans-2017-053264.29}

Introduction Multidrug-resistant N. gonorrhoeae infections are a threat to public health. In November 2015, UCLA Health began routine gyrase A (gyrA) genotyping all $N$. gonorrhoeae positive specimens, and reporting genotype and treatment recommendations for wild-type infections. Physicians were educated about wild-type gyrA genotypes predicting ciprofloxacin susceptibility. In May 2016 we began sending electronic reminders to providers of genotype results and treatment recommendations.

Methods We reviewed records for all laboratory confirmed $\mathrm{N}$. gonorrhoeae cases from January $1^{\text {st }} 2015$ - November $30^{\text {th }}$ 2016. Infections in different anatomic sites were considered unique infections, while unique infections in a single patient on the same date were considered a case. Empiric therapy was defined as treatment within one day of specimen collection.

Results Among 381 patients (32\% HIV infected) there were 411 cases and 459 anatomic site-specific N. gonorrhoeae infections. Of cases, 290 (71\%) were treated non-empirically. The average time to treatment among non-empirically treated cases $(\mathrm{n}=256)$ was 5.2 days (SD 4 days). After November 2015, there were 319 infections: 131 (41\%) were wild-type gyrA genotypes, $92(29 \%)$ mutant, 92 indeterminate and 4 were not attempted. Of the 92 indeterminate results 68 (74\%) were from the pharynx, compared to $24(26 \%)$ from other sites $(p$ value $<0.001)$. Among non-empirically treated cases, ceftriaxone was used in $119(96 \%)$ of 124 before versus $132(72 \%)$ of 184 after assay introduction ( $p$-value $<0.001)$. Among 59 non-empirically treated wild-type gyrA infections, 17 (29\%) were treated with ciprofloxacin; 2 (9\%) of 23 before electronic reminders began compared to $15(50 \%)$ of 30 after $(p$ value $=0.001)$, six cases had missing data. There was no ciprofloxacin use prior to assay implementation.

Conclusion A large health system successfully implemented routine $N$. gonorrhoeae gyrA genotyping with a reduction in ceftriaxone use. Targeted ciprofloxacin therapy increased with the use of electronic provider reminders.

\section{Oral Presentation Session 6}

\section{Host-Pathogen Interactions and Vaginal/ Urethral Microbiota}

\subsection{INVESTIGATING THE INTERACTION OF THE STEALTH PATHOGEN AND CAUSATIVE AGENT OF SYPHILIS, TREPONEMA PALLIDUM, WITH HUMAN PLATELETS}

${ }^{1}$ Brigette Church, ${ }^{2}$ John R Webb, ${ }^{1}$ Caroline E Cameron. ' University of Victoria, Victoria, Canada; ${ }^{2}$ BC Cancer Agency, Victoria, Canada

\subsection{6/sextrans-2017-053264.30}

Introduction Treponema pallidum ssp. pallidum, the causative agent of syphilis, is a highly invasive pathogen that interacts with a diverse repertoire of host cells during infection. The pathogen invades immunologically privileged sites and crosses the placental, blood-brain, endothelial and blood-retina barriers to establish widespread infection. Treponema pallidum disseminates via the circulatory and lymphatic systems, avoiding the prevalent inflammatory reactions raised against other bloodborne pathogens. In this study we investigate if T. pallidum uses an interaction with human platelets, key mediators of homeostasis and immune surveillance, to facilitate host persistence. We demonstrate that T. pallidum adheres to human platelets enabling survival for an extended period, and we discuss how this interaction may aid T. pallidum pathogenesis.

Methods Platelet rich plasma prepared from donor blood was incubated under host-mimicking microaerophilic conditions with viable T. pallidum, followed by examination for T. pallidum-platelet interactions via darkfield microscopy and flow cytometry analyses. Viability was confirmed using microscopic and fluorescent staining methodologies. 